\title{
Automatic dependent surveillance and broadcast
}

\section{ground-station optimal deployment problem}

\author{
N. Wang ${ }^{\mathrm{a}}$ D. Delahaye ${ }^{\mathrm{b}}$ A. Gondran ${ }^{\mathrm{c}}$ and M. Mongeau ${ }^{\mathrm{d}}$ \\ ENAC, Toulouse, France and \\ Université de Toulouse, France
}

\begin{abstract}
ADS-B (Automatic Dependent Surveillance and Broadcast) is a new surveillance technology that allows an aircraft to broadcast its own position periodically to ground stations. It has better precision, higher refresh rate, and lower cost than traditional secondary radar. Therefore, it is envisaged as a potential solution for air traffic surveillance in the context of nowadays growing traffic. In this study, we focus on a location problem that aims to deploy a network of ADS-B ground stations, in order to cover a given traffic space at minimum cost. We propose three mathematical optimization models based on a 2D discretized airspace under the form of discrete-optimization problems. Results obtained with the Mixed-Integer Linear Programming (MILP) solver Gurobi tested on a real-life size problem $(8,476$ daily flights over France) shows that our approach is viable in an operational context. The performances of the three proposed formulations are analyzed and compared.
\end{abstract}

\section{Introduction}

Automatic dependent surveillance-broadcast (ADS-B) is a new aeronautic surveillance technology that has been applied broadly around the world in recent years. ADS-B system consists of ground infrastructures and on-board transponders. This system provides two different services: ADS-B OUT and ADS-B IN. The ADS-B OUT service uses on-board transponders to broadcast

\footnotetext{
a PhD student, ENAC, 7 avenue Edouard-Belin, BP 5400531055 Toulouse cedex 4, France

b Professor, ENAC, 7 avenue Edouard-Belin, BP 5400531055 Toulouse cedex 4, France

c Assistant Professor, ENAC, 7 avenue Edouard-Belin, BP 5400531055 Toulouse cedex 4, France

d Professor, ENAC, 7 avenue Edouard-Belin, BP 5400531055 Toulouse cedex 4, France
} 
the aircraft position as well as other important flight information periodically down to the ground, or to other interested receivers such as communication satellites and other aircraft. The ADS-B IN service allows aircraft to receive ADS-B messages from nearby aircraft, and therefore to benefit from a clear understanding of the surrounding traffic situation. ADS-B OUT service has been well developed in practical applications, as installation of qualified transponders has been mandatory in several countries, while ADS-B IN service remains an operational choice for airline operators.

As an essential part of this system, ADS-B ground stations receive ADS-B OUT messages, process them, and then send these messages to Air Traffic Control (ATC) facilities, as showed in Fig. 1. Aircraft positions transferred by ADS-B are calculated based on a Global Navigation Satellite System (GNSS), which is more accurate and has much shorter refresh rate than traditional radar surveillance systems. ADS-B enables air traffic management systems to apply more efficient traffic routing procedures, empowers an increased capacity, and improves aviation safety. Additionally, an ADS-B ground infrastructure has lower installation and maintenance costs compared to radar systems. With these benefits, ADS-B technology has become a critical part of next-generation air traffic surveillance systems.

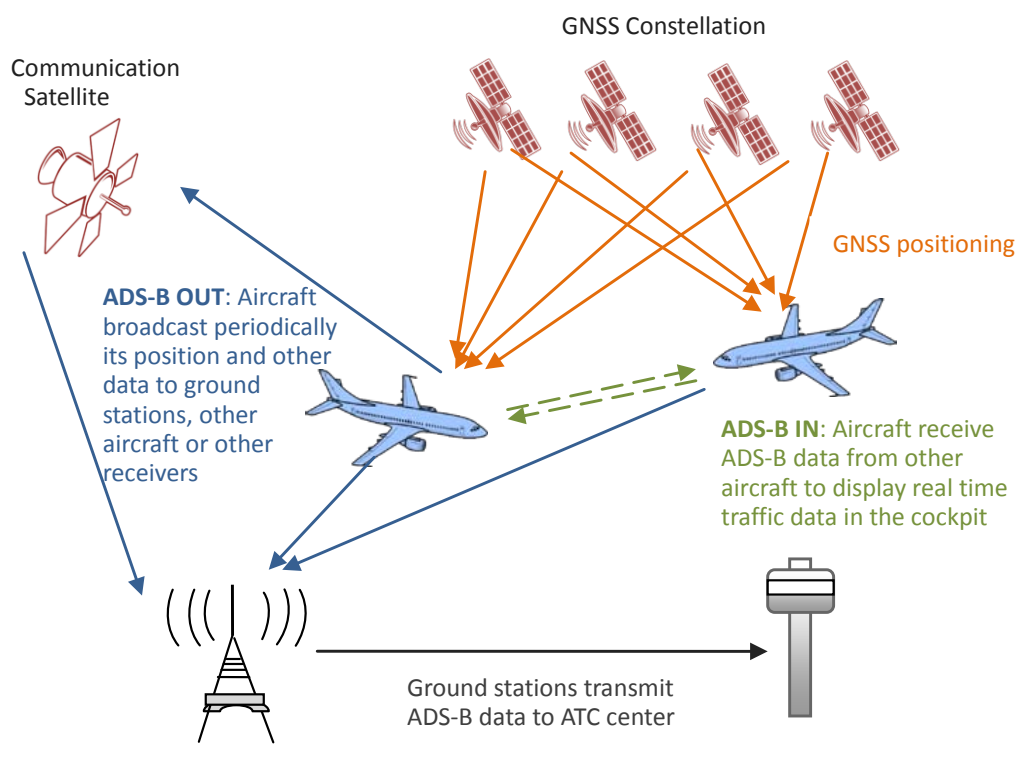

Fig. 1 ADS-B system

Up to now, several leading countries have started, or already finished the installation of ADS-B 
ground infrastructures. Australia started its ADS-B project in 2004. In 2009, it became the worldfirst for nationwide ADS-B coverage, with 28 sites throughout the country. In the United States, 634 sets of ADS-B ground facilities have been installed; a national-wide network was completed in 2014. This network provides ADS-B OUT services across the country for terminal and en-route service volumes, and it continues to grow to improve the service. In Europe, ADS-B deployment is progressing, combining with the Wide Area Multilateration (WAM) technology. About 70 ADS-B stations are currently deployed for operational use, and more than $700 \mathrm{WAM} / \mathrm{ADS}-\mathrm{B}$ stations are committed at various sites. In China, several ADS-B ground infrastructures have been deployed to cover separate west air routes, and more trial implementations are expected to continue the adaptation phase. Other ADS-B implementation related work is also being undertaken in all continents worldwide.

To deliver a stable and solid air traffic surveillance service, ADS-B ground stations have to be deployed across whole continents, including the old radar service areas and non-radar airspace. The layout of an ADS-B ground station network needs to be optimized to ensure a seamless coverage, and to meet air traffic safety requirements, while staying within a reasonable cost range. The present study contributes to the research in optimizing ADS-B ground station deployment by introducing three different mathematical formulations for the problem, and comparing their performances by solving the problem using a MILP solver, Gurobi [1].

\section{A. Previous related work}

To our knowledge, there is no exhaustive literature survey on nation-wide ADS-B deployment methodologies: no systematic approach for site selection has been yet proposed, and operational and geographical constraints are rarely taken into consideration in the ADS-B optimal deployment processes.

E. Boci [3-5] uses an integrated radio-frequency software for ADS-B coverage prediction. In his work, the siting solutions are proposed according to the maximum radar interference with the existing radar sites; and for the non-radar areas, the cone-of-silence problem due to the vertical pattern of ADS-B antenna is addressed in order to determine the distance between two sites. These siting principles allow the author to implement a computer algorithm for searching some initial 
solutions to cover the entire US territory.

A more recent work, by W. Langhans et al. [8], presents a national-wide WAM system for Austria, which consists of one central processing station and 61 remote units. Apart from taking in account a multiple-coverage requirement, this network must also be synchronized to perform multilateration. A site-scoring system is presented in this work to perform the site selection. Performance requirements such as position accuracy, air-route coverage, and data capacity are used as metrics for guiding the optimization process.

W. Zhang [10] gives a detailed analysis of ADS-B ground-station range calculation and visual field calculation based on Geographical Information System (GIS) data in order to realize seamless coverage of few air routes in western China. Similar research work on radar or sensor networks can also provide useful guidelines for research on this subject, see for instance [7, 9].

\section{B. Overview}

The present study focuses on large-scale ADS-B ground-station optimal deployment, concentrating more precisely on a nation-wide integrated ADS-B ground station network. To ensure an efficient and robust ADS-B function service, we concentrate particularly on the mathematical formulation of the problem. The results are expected to provide reasonable initial deployment plans to decision makers.

The remainder of this article is organized as follows: Section 2 analyses ADS-B ground station performance to define the coverage range. Section 3 introduces three mathematical optimization models based on a 2D discretized airspace. Numerical tests based on real traffic data are performed, and results obtained by Gurobi are presented in Section 4. Finally, Section 5 presents a conclusion on this study and draws perspectives.

\section{ADS-B ground-station performance}

The antenna of an ADS-B ground-station can receive messages sent from the on-board transponder within a certain distance, called the station coverage range, noted $R$. This range depends on many factors: the transmitted-signal frequency and strength, the sensibility of the receiving antenna, the propagation loss during the transmission path, etc. 
ADS-B data link uses Very High Frequency (VHF) electromagnetic waves. The wave follows the Line Of Sight (LOS) propagation rule, which means that in perfect vacuum, it travels following straight line. When signals transfer from aircraft to the ground or the opposite, the curvature of Earth affects the sight propagation line (radio horizon effect). Remark that the coverage range $R$ is smaller than the maximum LOS range, $d_{L O S}$, the farthest propagation distance of the sight line, as showed in Fig. 2, where $R_{E}$ denotes the mean radius of the Earth.

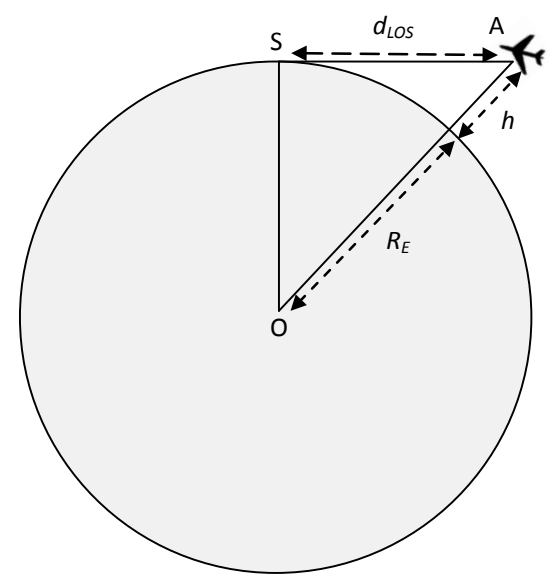

Fig. 2 LOS range from an antenna site $\mathbf{S}$ to an aircraft $\mathbf{A}$ at height $h ; O$ and $R_{E}$ denote respectively the center and the mean radius of the Earth.

Suppose that Earth is a perfect sphere and that there is no atmosphere, then an approximate LOS range can be calculated by a simple formula. Given the mean radius of Earth $R_{E}=6,371 \mathrm{~km}$, and the height of the aircraft in flight $h$, if the height of the station is neglected, the LOS range is expressed as:

$$
d_{L O S}=\sqrt{\left(R_{E}+h\right)^{2}-\left(R_{E}\right)^{2}}
$$

In the usual case, since $h \ll R_{E}$, the above formula can reasonably be approximated by:

$$
d_{L O S}=\sqrt{2 R_{E} h}
$$

With the height $h$ given in meters, and $d_{L O S}$ in kilometers, we get:

$$
d_{L O S}[k m] \approx 3.57 \sqrt{h[m]}
$$


The geodetical distance $d_{G E O}$ is the distance measured along the surface of the Earth:

$$
d_{G E O}=R_{E} \arccos \left(\frac{R_{E}}{R_{E}+h}\right)
$$

For the range of our data, the maximum geodesic distance and the maximum LOS range are approximately equal: $d_{G E O} \approx d_{L O S}$. Then, in first approximation, the coverage range $R$ is equal to $d_{L O S}$. However, in reality, under the refractive effect of atmosphere, VHF radio waves actually travel as a curve. Meanwhile, the atmosphere absorbs partially the wave energy during the propagation. Other environment aspects, like multi-path effect, can also decrease the effective propagation distance. Taking the above factors into account, a list of maximum coverage $R \approx 0.872 \times d_{L O S}$ for representative Flight Levels (FL) is presented in Table 1.

Table 1 Maximum coverage range for different flight levels

\begin{tabular}{|c|c|c|c|c|c|}
\hline FL (100 feet) & 30 & 120 & 240 & 330 & 400 \\
\hline Corresponding altitude $h(\mathrm{~m})$ & 914 & 3657 & 7315 & 10058 & 12192 \\
\hline$d_{L O S} \approx d_{G E O}(\mathrm{~km})$ & 108 & 216 & 305 & 358 & 394 \\
\hline Maximum coverage $R(\mathrm{~km})$ & 94 & 188 & 266 & 312 & 344 \\
\hline
\end{tabular}

Besides the electromagnetic wave attenuation, terrain blockage is another important factor to take into account when analyzing the effective coverage field of an antenna. Fig. 3 shows the influence of a geographical obstacle, for example mountains, on antenna coverage from a vertical view (a) and a plan view (b). The vertical profile of the antenna coverage depends on the shadowing angle of the obstacle, $\theta_{s}$. 

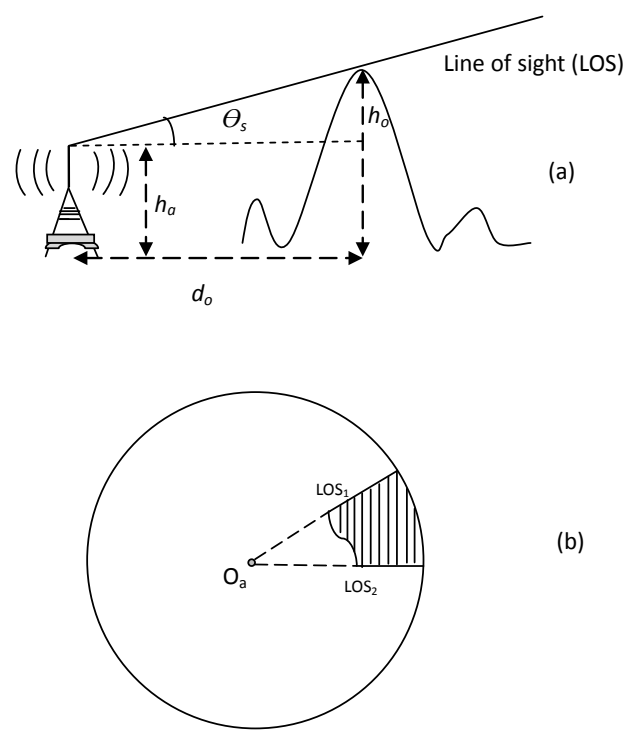

(b)

Fig. 3 Terrain blockage: vertical (a) and plan (b) views. The shadowing angle of the obstacle is given by: $\tan \theta_{s}=\frac{h_{o}-h_{a}}{d_{o}}$

Effective Coverage Field (ECF) analysis relies on detailed GIS data and on a Digital Elevation Model (DEM). Realizing seamless coverage with ECF of all candidate station sites therefore first involves obtaining an ECF database. This database comes normally from a pre-calculation performed by specialized software $[3,4,10]$. The challenge here is to design a dynamic algorithm that can deal with real-time ECF computations integrated within the optimization process. This requires much memory space and a powerful processor. Such an approach has the advantage of allowing the decision maker to consider a continuum of candidate site positions, instead of a finite set of given discrete positions. In this article, we do not take into account of ECF and discrete candidate site positions is considered.

\section{Mathematical formulations of the problem}

In this section, we first introduce the airspace discretization model to process the real traffic data. Then, we formulate our ADS-B ground-station deployment problem under the form of a classical Set Covering Problem (SCP). According to the different considerations of the decision maker, two other formulations are also proposed for this problem: the second formulation accepts a 
flexible trade-off between full-coverage and the cost associated to a high number of selected stations, while the third formulation maximizes the coverage with a fixed number of selected stations.

\section{A. 2D airspace discretization}

Here we consider the airspace as the 2D projection of a given 3D flight zone; all civil aviation trajectories inside this zone are also projected in 2D. Consider a rectangle large enough to include this airspace; this rectangle is then discretized as a grid $\left(G_{a}\right)$ consisting of small squares, called grid elements, of size $a_{s} \times a_{s}$, where $a_{s}$ is the airspace step size.

In this preliminary study, we assume that the coverage range of all station are identical, noted as $R$. Hence, the coverage field of any station is a $R$-radius disk when terrain blockage is not taken into account. A grid element $i$ is considered to be covered by a station if the distance between the central point of this grid element and the station position is less than or equal to $R$, as illustrated in Fig. 4. In further works, we integrate ECF in order to take into account the terrain blockage as shown in Fig. 3-b. We consider that a set of candidate ADS-B station sites is provided as input to the problem. For this study, we shall generate artificially a set of candidate stations by using some other discretization grid of the airspace $\left(G_{c}\right.$ with candidate-station step size $\left.c_{s}\right)$ and assuming that there is a candidate station site at the center of each grid element generated thereby.

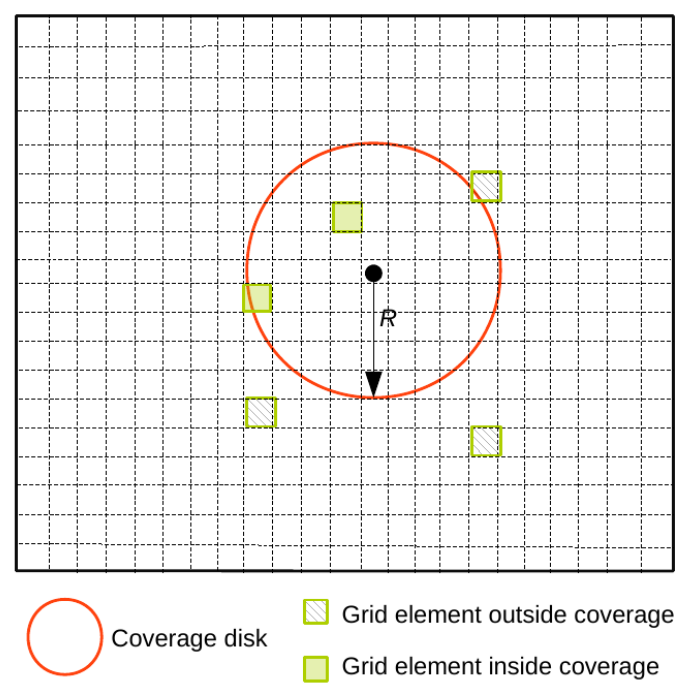

Fig. 4 2D airspace discretization and coverage field of one ADS-B station 


\section{B. Traffic density}

In order to generate a realistic instance of the ADS-B ground station optimal deployment problem, we use real-traffic data to create a traffic density map based on our discrete airspace. The traffic data consists of 4D trajectory sample points (latitude, longitude, altitude, time). These points are then projected on the $2 \mathrm{D}$ grid of our discretized airspace $G_{a}$. Each grid element $i$ has a density index $d_{i}$, defined as the total number of trajectory sample points lying in the grid element $i$. Grid elements with higher density index can thereby be given higher priority during the optimization phase. Fig. 5 shows an example of 50 simulated direct flights in France. Fig. 6 displays the correspondent density map; grid elements featuring darker colors correspond to higher density indices.

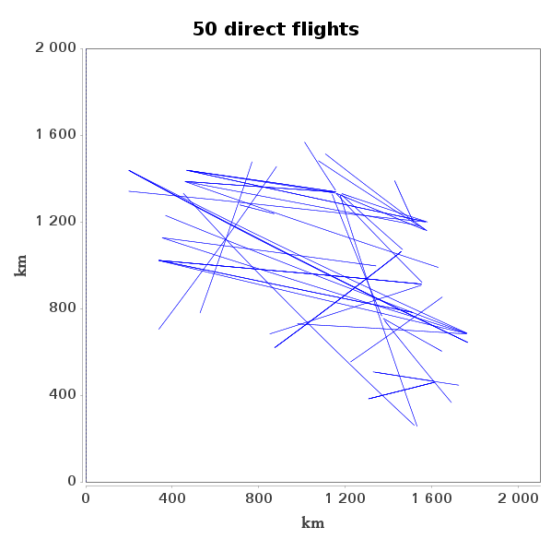

Fig. 550 direct flights

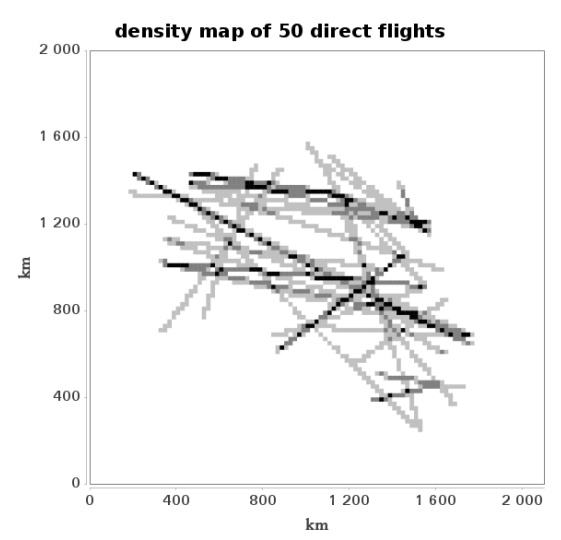

Fig. 6 Corresponding density map

\section{Optimization formulations}

This sub-section presents three optimization formulations for the ADS-B ground station optimal deployment problem. Recall that in this study, we assume that candidate stations have identical coverage ranges and identical installation costs. These hypotheses imply that minimizing the cost of an ADS-B network is equivalent to minimizing the number of selected ground stations.

With the 2D airspace discretization, instead of constructing an ADS-B ground station network to cover every sample point of each flight trajectory, we aim at covering all the non-empty grid elements (whose density index is not zero). We first consider a basic, single-layer coverage problem, where each grid element must be covered at least once. Under the special context of air-traffic 
surveillance, additional coverage performance requirements can be added. More precisely, in the robust version of the problem, which aims at delivering a service that is robust to material failures, station redundancy is required. This means that each grid element needs to be covered by at least two stations, so that in the occurrence of one station out of mission, there is another one to complete the service. Another practical motivation for considering the multi-layer coverage problem is the multilateration application, for which each grid element needs to be covered by at least three stations for allowing $2 \mathrm{D}$ position calculations, and four stations for $3 \mathrm{D}$ position calculations to ensure the proper implementation of this cooperative aircraft surveillance technology. These extra coverage requirements can easily be translated into inequality constraints in the optimization formulations we are about to present.

The first optimization formulation we present here aims at selecting a minimal number of stations, while ensuring that every grid element is covered by at least $K$ stations. This optimization problem can be modeled as a Set Covering Problem (SCP), which is the most-common optimization model in facility location problems [6].

Let us define the following notation:

- Given input data:

$-E=\{1,2, \ldots, n\}$ is the set of non-empty grid elements; $i \in E$ will designate a specific grid element

$-S=\{1,2, \ldots, m\}$ is the complete set of candidate stations; $j \in S$ will designate a specific candidate station

$-K \in \mathbb{N}^{+}$is the required coverage level: $K=1$ in the single-layer coverage version of the problem, $K=2$ in the robust variant, and $K=4$ in the multilateration application

- $S_{i}$, denotes the set of candidate stations that can cover grid element $i, i \in E$

- Optimization variables:

$-x_{j}$ is a binary decision variable whose value is 1 if the candidate station $j$ is selected, 0 otherwise, $j \in S$ 
The first formulation of our problem (SCP), is the following Integer Linear Program (ILP):

$$
\begin{gathered}
\underset{x}{\operatorname{minimize}} \\
\sum_{j=1}^{m} x_{j} \\
\text { subject to } \\
\sum_{j \in S_{i}} x_{j} \geq K, i=1,2, \ldots, n \\
x_{j} \in\{0,1\}, j=1,2, \ldots, m
\end{gathered}
$$

where $x$ is the vector whose $j^{t h}$ component is the binary decision variable $x_{j}$.

The SCP is a well-known combinatorial optimization problem. It is NP-hard in the general sense, but with the fast development of commercial solvers, even large instances can be solved to optimality with exact methods. One may consider for instance, the commercial optimization solver Gurobi [1]. It relies on a branch and cut method to solve ILP problems, while using heuristic approaches to generate efficient cutting planes and admissible solutions.

Heuristic algorithms can also be considered to tackle large instances of the SCP, in which the constraint of full coverage usually raises difficulties. Indeed, our empirical tests with basic heuristic approaches involving local-improvement steps exhibit premature convergence towards local optima that are not satisfactory. A natural idea consists in allowing the search to proceed temporarily outside the feasible domain. This, in return, involves addressing the very difficult task of projecting the current infeasible iterate back into the feasible domain without losing too much quality in terms of the objective-function value.

Therefore, we propose a reformulation of the problem that penalizes constraints (2) by adding a term in the objective function. Inspired by the reformulation proposed by Bilal et al. [2], our original SCP formulation becomes what we call the penalized formulation:

$$
\begin{aligned}
& \underset{X=(x, y)}{\operatorname{maximize}} \quad f(X)=\sum_{i=1}^{n}(1+\epsilon) y_{i}-\sum_{j=1}^{m} x_{j} \\
& \text { subject to } \\
& y_{i}=\min \left(\sum_{j \in S_{i}} x_{j}, K\right), i=1,2, \ldots, n \\
& x_{j} \in\{0,1\}, j=1,2, \ldots, m \\
& y_{i} \in\{0,1, \ldots, K\}, i=1,2, \ldots, n
\end{aligned}
$$

- $y_{i}$ is an auxiliary integer-valued decision variable that is related to the violation level of the 
specific constraint in (2) that corresponds to the grid element $i, i \in E$

- $X$ denotes the vector of optimization variables: $(x, y)$, where $y$ is the vector whose $i^{t h}$ component is $y_{i}, i \in E$

- $\epsilon$ is a user-defined parameter

Constraints (5) define the link between the vectors of decision variables $x$ and $y$ : since we only need to reach the given target coverage level $K$, when a grid element $i \in E$ is more covered than this required level, the extra service provided is considered useless, therefore it is not taken into account in $y_{i}$. In other words, once a grid element reaches a coverage level higher than required, it does not contribute further to the total-coverage objective-function value.

The sign of the user-defined parameter $\epsilon$ plays an important role in this reformulation. From the decision maker's point of view, if the full-coverage is a hard constraint (that must be satisfied), then $\epsilon$ should be given a strict positive value. In this case, the coverage constraints (2) are enforced and the above penalized reformulation is equivalent to the SCP formulation. Indeed, by generalizing the results of [2], we have:

Proposition 1 Assuming that there exists a feasible solution for the SCP formulation, the SCP formulation and the penalized formulation are equivalent optimization problems in the case where $\epsilon>0$. More precisely: an optimal solution of one problem can straightforwardly yield an optimal solution for the other problem.

Proofs: see Appendix A.

When $-1<\epsilon \leq 0$, the penalized formulation is a relaxation of the original SCP formulation. If full-coverage can be compromised, one should choose a value in $]-1,0]$. In this case, the penalized reformulation boils down to optimizing the weighted sum of two criteria: maximizing the sum of coverage for each grid element, while minimizing the number of selected stations. For example, when $K=1$ and $\epsilon=-0.9$, any extra grid elements covered yields an increase of $1+\epsilon=0.1$ in the first criterion (coverage), and an increase of 1 in the other criterion (number of stations). As a result, the objective-function value is changed by $0.1-1=-0.9$. One can then easily see that one extra station must cover at least 10 grid elements that are not covered yet to obtain an increase in 
the objective-function value.

In order to allow the possibility of presenting a pool of good alternate solutions to the decider, we propose a third optimization formulation: the maximum covering $S C P$ (MSCP) formulation. In this formulation, we fix the number of stations to some pre-defined value $N$ and we look for the $N$ stations that cover as many grid elements as possible:

$$
\begin{aligned}
& \operatorname{maximize}_{X=(x, y)} \quad \sum_{i=1}^{n} y_{i} \\
& \text { subject to } \\
& y_{i}=\min \left(\sum_{j \in S_{i}} x_{j}, K\right), i=1,2, \ldots, n \\
& \sum_{j=1}^{m} x_{j}=N \\
& x_{j} \in\{0,1\}, j=1,2, \ldots, m \\
& y_{i} \in\{0,1, \ldots, K\}, i=1,2, \ldots, n
\end{aligned}
$$

\section{Numerical tests}

In this section, we first present the traffic data used for the numerical tests in Subsection 4.1. The next two subsections present and compare the results obtained with the original SCP formulation and the penalized formulation with $\epsilon>0$ (Subsection 4.2), and with the MSCP formulation and the penalized formulation with $\epsilon<0$ (Subsection 4.3). All tests are carried out with the MILP solver Gurobi version 7.2 on an Intel i7-4700 2.4GHz processor with 8 cores.

\section{A. Traffic data}

We test our problem on a large realistic instance constructed from data of flight plans corresponding to a period of 24 hours over the France airspace; it involves 8,476 flights, displayed on Fig. 7. The data is provided under the form of a table of $1,874,741$ trajectory sample points, resulting from a time discretization with a time step of 15 seconds. A square of 2,000 $\mathrm{km} \times 2,000$ $\mathrm{km}$ that includes the French territory is taken as the 2D airspace. This 2D airspace is discretized into a grid $G_{a}$ with step size of $a_{s}=20 \mathrm{~km}$. After projecting the trajectory sample points in this 2D grid, we obtain an instance involving 10,000 square grid elements. To generate the set $S$ of candidate stations, we use the discretization step size of $c_{s}=10 \mathrm{~km}$, that yields 40,000 candidate 
stations. We then use a pre-processing algorithm to eliminate the empty grid elements (those not intersecting any trajectory) and the candidate stations that do not cover any grid element. As a result, the instance has $n=2,700$ non-empty grid elements and $m=6,687$ candidate stations. In this preliminary study, we suppose that all candidate station radius are identical and taken to be $R=200 \mathrm{~km}$ (which corresponds to consider all flights at FL 110), and they feature identical installation costs.

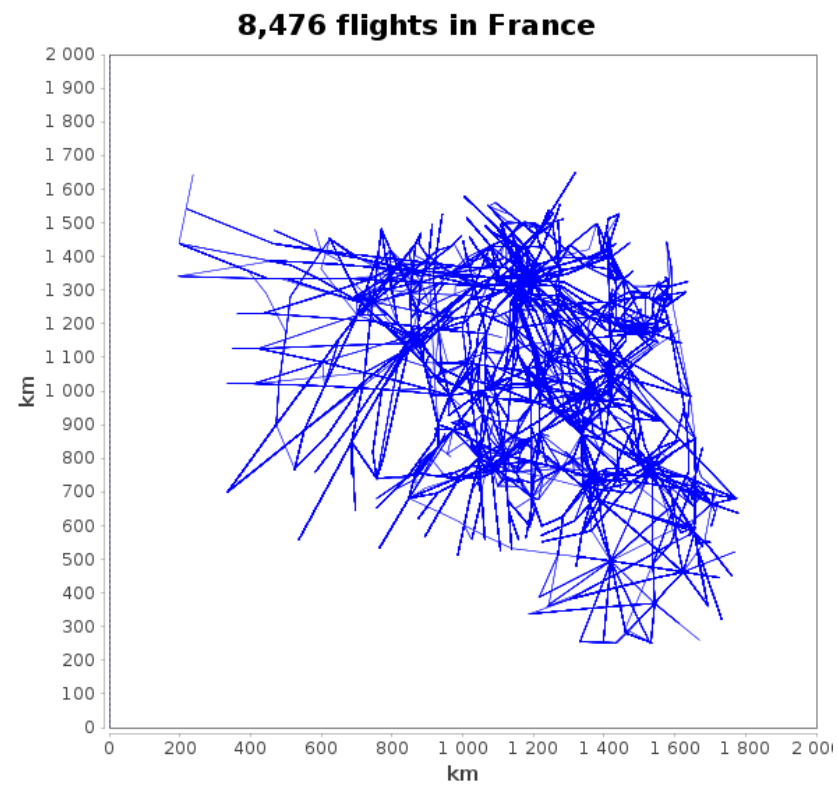

Fig. 7 One day of air traffic in France: 8,476 flights

\section{B. Exact covering: comparison of the SCP formulation and the penalized formulation with} $\epsilon>0$

First, we study the case of exact covering that we modeled with the SCP formulation and the penalized formulation with $\epsilon>0$ and that requires all the grid elements to be covered by at least $K$ ADS-B antenna.

Results obtained by Gurobi are presented in Table 2, where $N^{*}$ is the minimal number of selected stations obtained for different $K$ values; $t_{\mathrm{SCP}}$ and $t_{\epsilon>0}$ are respectively the CPU run time in seconds for the original SCP and the penalized formulations. The SCP formulation clearly dominates the penalized formulation with $\epsilon>0$.

The SCP formulation always yields an optimal solution (but with very large CPU times when 
$K=4$ et $K=5$ ). The penalized formulation with $\epsilon>0$ yields faster convergence than the SCP formulation only when $K=1$, but it does not succeed to find an optimal solution within 48 hours of CPU time when $K=4$ et $K=5$. The optimal number of stations displays almost perfect linear increase with $K$ values, as showed in Fig. 8. This reveals that for each $K$ coverage, there are only a limited number of grid elements exceeded the requirement coverage level. For example, 17 stations are required for $K=1$, if each grid element is covered only once, then 17 more stations are required for $K=2$; but in reality, the coverage area of the 17 stations overlapped for a number of grid elements so that only 16 more stations are required for $K=2$.

Table 2 Optimal number of stations and CPU times (in seconds) obtained from the SCP formulation and the penalized formulation with $\epsilon>0$ for different $K$ coverages (" -" means over 48 hours of CPU time).

\begin{tabular}{lccccccc}
\hline \hline$K$ & 1 & 2 & 3 & 4 & 5 & 6 & 7 \\
\hline$N^{*}$ & 17 & 33 & 49 & 64 & 80 & 96 & 112 \\
$t_{\mathrm{SCP}}$ & 103 & 547 & 6,042 & 42,511 & 25,722 & 4039 & 4282 \\
$t_{\epsilon>0}$ & 90 & 2,746 & 13,716 & - & - & 7146 & 5801 \\
\hline \hline
\end{tabular}

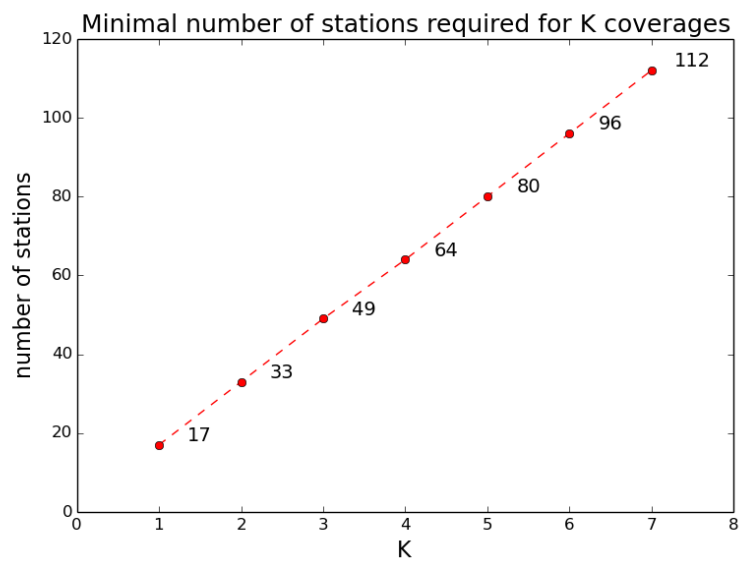

Fig. 8 Minimal number of stations needed for different $K$ coverages

The optimal solution obtained from the single-covering $(\mathrm{K}=1)$ instance is displayed in Fig. 9, where the points represents the non-empty grid elements while each green circle represents the coverage disk of one selected station. All grid elements are covered by these $N^{*}=17$ selected 
stations.

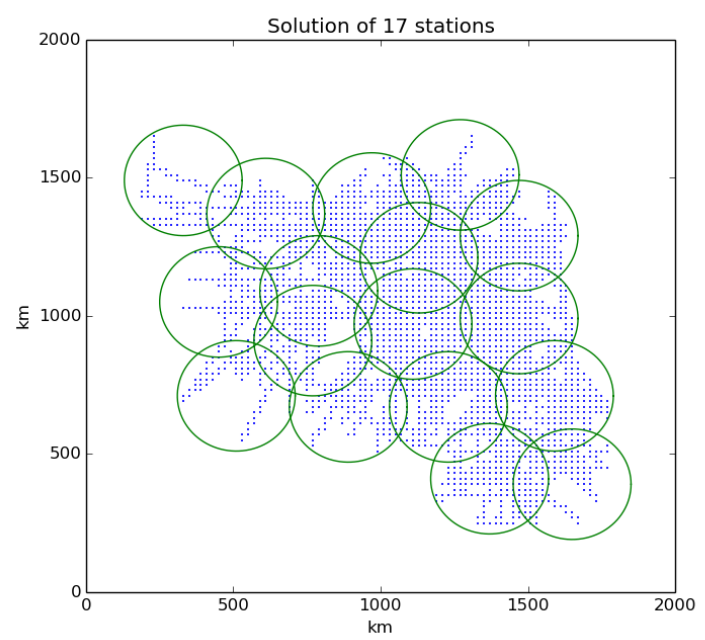

Fig. 9 Deployment of the $N^{*}=17$ stations with covering $(K=1)$ the $\mathbf{8 , 4 7 6}$ flights over France. The points represent the 2,700 grid elements, each green circle represents the coverage disk of one of the 17 selected stations.

C. Partial covering: comparison of the MSCP formulation and the penalized formulation with $\epsilon<0$

In this Subsection, we study ADS-B deployment when one allows some grid elements not to be covered at the required level. This corresponds to the MSCP formulation and the penalized formulation with $\epsilon<0$.

Given a set of selected stations, let us define the overage deficit of the grid element $i, i \in E$ to be $d c_{i}:=K-y_{i}=K-\min \left(\sum_{j \in S_{i}} x_{j}, K\right)$, and let $d c$ be the total coverage deficit: $d c:=\sum_{i=1}^{n} d c_{i}$. Results obtained by the MSCP formulation for various input values for the desired number $N$ of selected stations are presented in Table 3. To solve these instances, we use Gurobi with a time limit of 2 hours (7200 seconds). Global optima proved by Gurobi are noted in bold in the table, otherwise only the suboptimal solution (upper bound) and the lower bound found are provided. In this table, $N$ denotes the fixed number of stations, "best $d c$ " denotes the best value of $d c$ found by Gurobi at the end of two hours, "LB $d c$ " denotes the lower bound of $d c$ found by Gurobi at the end of two hours. Remark that for each value of $\mathrm{K}$, the first value of $N$ is the optimal numbers of selected stations found previously by the SCP formulation (for which full coverage is thereby 
possible). In the case where $K=1,2$ and 3 , MSCP formulation proves the optimality faster than SCP formulation. For $K=4$ and $N=64$, the MSCP do not prove the optimality in 2 hours.

Table 3 Coverage deficit obtained by Gurobi from the MSCP formulation for $K=1,2,3$, and 4 for various input values for the desired number $N$ of selected stations.

\begin{tabular}{|c|c|c|c|c|c|c|c|c|c|c|}
\hline \multirow{4}{*}{$K=1$} & $N$ & 17 & 16 & 15 & 14 & 13 & 12 & 11 & 10 & 9 \\
\hline & best $d c$ & 0 & 3 & 17 & 45 & 83 & 144 & 217 & 331 & 464 \\
\hline & $\mathrm{LB} d c$ & 0 & 3 & 17 & 34 & 83 & 144 & 217 & 331 & 464 \\
\hline & time $(\mathrm{s})$ & 12 & 3766 & 5218 & 7200 & 5952 & 5670 & 2079 & 677 & 420 \\
\hline \multirow{4}{*}{$K=2$} & $N$ & 33 & 32 & 31 & 30 & 29 & 28 & 27 & 26 & 25 \\
\hline & best $d c$ & 0 & 3 & 16 & 34 & 61 & 87 & 124 & 160 & 214 \\
\hline & $\mathrm{LB} d c$ & 0 & 0 & 7 & 19 & 38 & 65 & 97 & 143 & 198 \\
\hline & time $(\mathrm{s})$ & 69 & 7200 & 7200 & 7200 & 7200 & 7200 & 7200 & 7200 & 7200 \\
\hline \multirow{4}{*}{$K=3$} & $N$ & 49 & 48 & 47 & 46 & 45 & 44 & 43 & 42 & 41 \\
\hline & best $d c$ & 0 & 4 & 13 & 23 & 43 & 64 & 90 & 121 & 156 \\
\hline & $\mathrm{LB} d c$ & 0 & 0 & 4 & 13 & 27 & 44 & 68 & 97 & 121 \\
\hline & time $(\mathrm{s})$ & 118 & 7200 & 7200 & 7200 & 7200 & 7200 & 7200 & 7200 & 7200 \\
\hline \multirow{4}{*}{$K=4$} & $N$ & 64 & 63 & 62 & 61 & 60 & 59 & 58 & 57 & 56 \\
\hline & best $d c$ & 4 & 10 & 25 & 31 & 53 & 70 & 96 & 125 & 165 \\
\hline & $\mathrm{LB} d c$ & 0 & 4 & 10 & 22 & 35 & 52 & 72 & 97 & 124 \\
\hline & time $(\mathrm{s})$ & 7200 & 7200 & 7200 & 7200 & 7200 & 7200 & 7200 & 7200 & 7200 \\
\hline
\end{tabular}

In fact, the number of stations $N$ and the total coverage deficit $d c$ are two contrary objectives for our problem (that have both to be minimized). The problem is fundamentally bi-objective. Fig. 10 displays two graph that show two Pareto front (best trade-offs) corresponding to the solutions found by the MSCP formulation (in blue) and the penalized formulation with $\epsilon<0$ (in red) for $K=1$ (left) and $K=4$ (right). The solutions obtained for the MSCP formulation results from providing various values for the input parameter $N$ (imposed number of stations to be selected). On the other hand, the solutions of the penalized formulation (with $\epsilon<0$ correspond to different settings of the input penalty parameter $\epsilon$, according to the emphasis the decider wishes to place on minimizing the number of stations (cost) rather than minimizing the lack of coverage. The two populations of solutions are close but, the penalized formulation with $\epsilon<0$ finds less interesting results with the 
same number of selected stations: it takes longer computation time than the MSCP formulation to find the same results; or it ends up with more grid elements not covered by the selected stations at the end of two hours of CPU time. There is only one exception: for $K=4$ and $N=56$ the penalized formulation with $\epsilon<0$ finds a solution with $d c=161$, while the MSCP formulation ends up with $d c=165$.
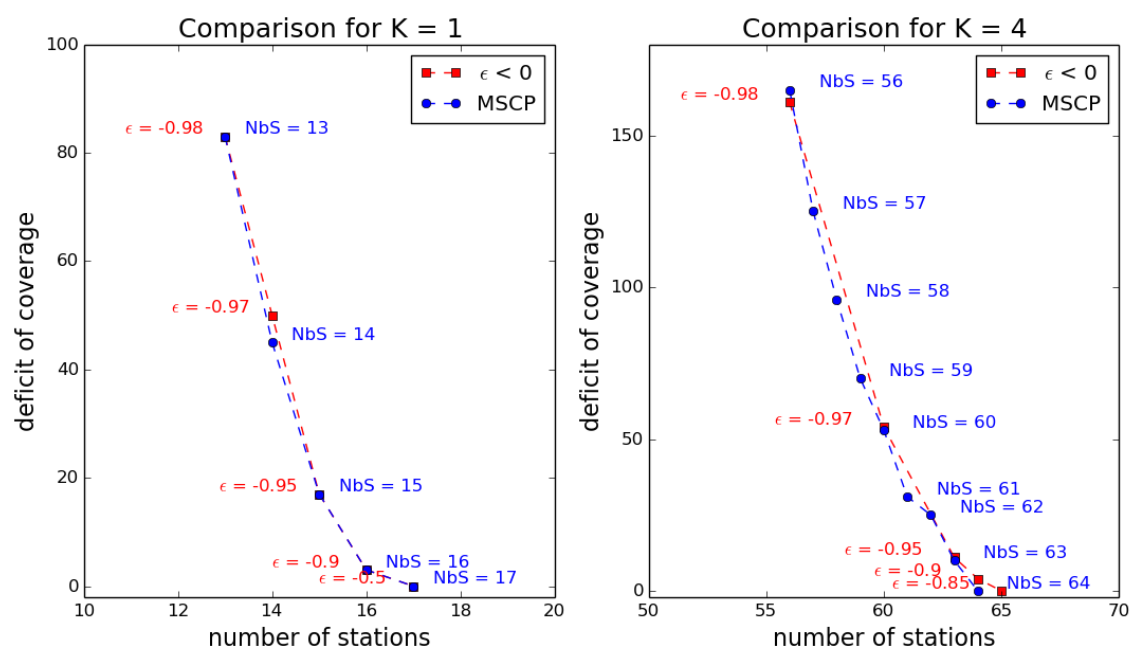

Fig. 10 Comparison of the MSCP and the penalized $(\epsilon<0)$ for $K=1$ and $K=4$.

Fig. 11 (respectively Fig. 12) represents an example of partial covering found with the MSCP formulation with $K=1$ and $N=16$ (resp. with $N=15$ ). Green circles represents the coverage disk of each selected station, the blue points represent the grid elements covered by the selected stations, and the red points represent the grid elements not covered. Remark that, apart for one grid element, all the grid elements not covered are in fact located on the outer edge of the airspace, actually outside the French land borderlines. A complete solution pool consisting of different trade-offs between the two criteria (cost and coverage) is presented in Fig. 13. Such a multicriteria decision-aid point of view may be of interest in practical applications. 

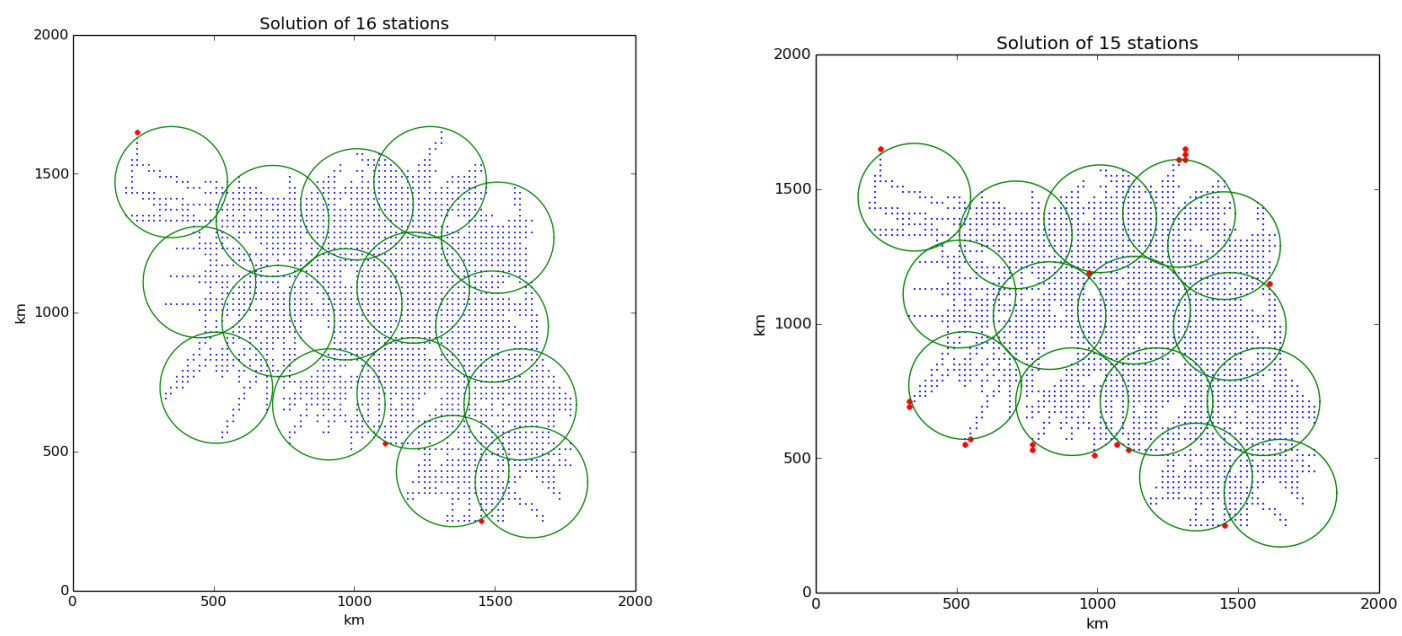

Fig. 11 Deployment of 16 stations with 3 grid Fig. 12 Deployment of 15 stations with 17 grid elements not covered. elements not covered.

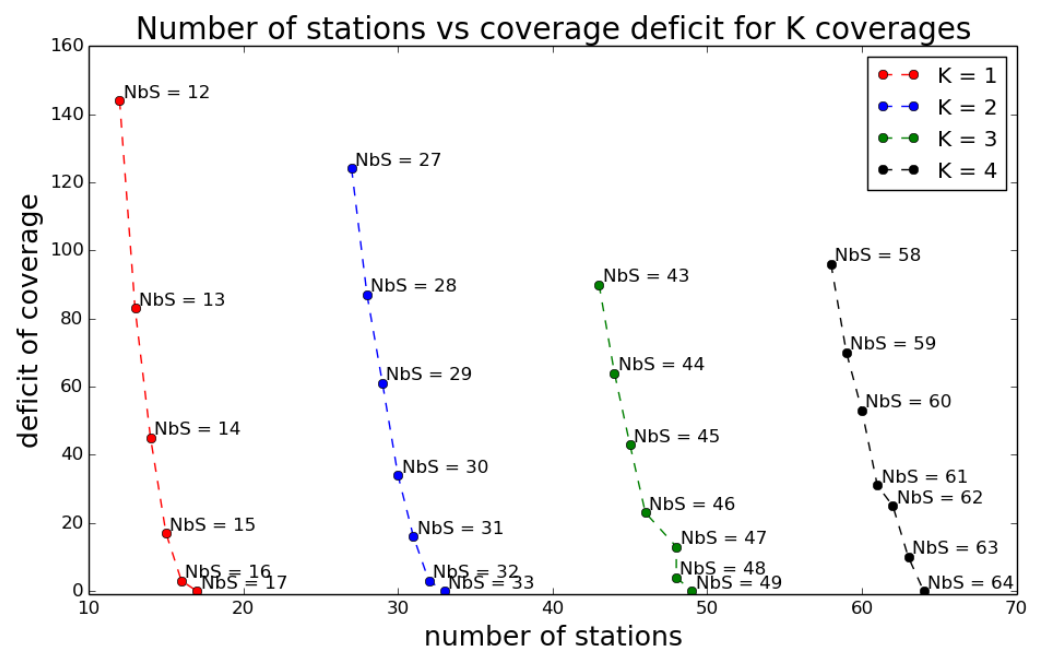

Fig. 13 Number of stations $(N)$ vs total coverage deficit $(d c)$ for different $\mathbf{K}$ coverages.

\section{Conclusion and perspectives}

In this paper, we introduced a specific facility location problem for the coming aviation surveillance technology ADS-B. Based on a discretized 2D airspace model, we first formulated this problem as the classical operations-research Set Covering Problem (SCP) with multi-coverage: each aircraft must send messages to $K$ ADS-B antennas, where $K$ takes value from 1 up to 7 . Next, we considered the application context in which the decision maker must trade off between the number of 
ADS-B stations to deploy (cost) and the coverage level, for instance in the robust or mulilateration problems. We thereby proposed a penalized formulation, inspired by the work of Bilal et al. [2] initially proposed for the single-coverage $(K=1)$ case that we extended to the multi-coverage case. The last model we proposed, the maximum set covering problem formulation, allows the user to impose the number of stations to be deployed in his search for compromised solutions.

In order to test and compare the above models, we build a real-life size problem instance involving 8,476 flights, from real traffic data in the France airspace. We presented results obtained with the state-of-the-art MILP solver Gurobi. Optimal solutions were found for a series of different coverage requirements. These results show that our approach is viable in an operational context and the MSCP formulation contributes a lot to build the solution pool for decision makers in the end. While the performance of penalized formulation is not as good as the other two formulations in the simplified context of this preliminary study, but it may be more effective than the other two formulations when more realistic features will be considered. Among such features that can be interested in the penalized model, let us mention using a 3D model taking into account the elevation of station position and the terrain blockage around the position (caused by obstacles such as mountains) that will replace the $2 \mathrm{D}$ discretized airspace model. This will require the integration of geographical information into the current model.

Covering problems have basically two criteria to optimize: on one hand, the cost of the solution, evaluated by the number of installed stations and our models can easily account for installation costs that differ from one station to another. On the second hand, the quality of service (QoS) of the solution, evaluated by the number of clients here, the trajectory points obtained by discretization (sufficiently) covered. In our specific case, the QoS is not only the number of ADS-B antenna that cover each point of the airspace, like in this study, but rather a more complex evaluation of the QoS based on the possibility to localize one aircraft in the airspace by trilateration. For example, if some of the four antenna covering a given airspace point are localized close from each other, then the trilateration is subject to large error. This leads to interesting perspectives in the continuation of our work:

- A combination of wide-area mulilateration technology and ADS-B service is promising in 
the future: in this case, each trajectory point has to be covered by at least four stations and distances and angles between these four stations need to be carefully restricted via constrained optimization models in order to ensure accuracy of the calculation of the aircraft position.

- In the context of more complex QoS measurement, non-linear optimization should be taken into account. As a consequence, specific heuristics methods based on the penalized formulation should be considered, instead of the exact linear Gurobi solver, in order to address the resulting difficult optimization problems.

\section{Acknowledgements}

This work has been supported by the Civil Aviation University of China (Tianjin) and the China Scholarships Council program.

Appendix: Proof of equivalence for the first two formulations

Proposition 1 Assuming that there exists a feasible solution for the SCP formulation, the SCP formulation and the penalized formulation are equivalent optimization problems in the case where $\epsilon>0$. More precisely: an optimal solution of one problem can straightforwardly yield an optimal solution for the other problem.

Proofs: First, remark that an optimal solution $X^{*}=\left(x^{*}, y^{*}\right)$ of the penalized formulation can be truncated into a solution $x^{*}$ that is an optimal solution for the SCP formulation. Indeed, in a first step, we prove that this solution $x^{*}$ is feasible for the SCP formulation. Suppose by contradiction that $x^{*}$ were not feasible for the SCP formulation. This means that there exists at least one grid element $I \in E$ that does not reach its target coverage level $K$. Since the SCP formulation is feasible, there exists at least one non-selected station $J \in S$ that covers the element $I$. Consider the following solution $\bar{X}$ for the penalized formulation so that it is identical to $X^{*}$ except that it includes moreover the station $J$ (more precisely, $\bar{x}_{J}:=x_{J}^{*}+1, \bar{x}_{j}:=x_{j}^{*}$ for all $j \neq J ; \bar{y}_{I}:=\min \left(\sum_{j \in S_{I}} \bar{x}_{j}, K\right)$, 
$\bar{y}_{i}=y_{i}^{*}$ for all $\left.i \neq I\right)$. Its objective-function value is

$$
\begin{aligned}
f(\bar{X}) & =f\left(X^{*}\right)+(1+\epsilon)\left(\bar{y}_{I}-y_{I}^{*}\right)-1 \\
& =f\left(X^{*}\right)+(1+\epsilon)-1 \\
& =f\left(X^{*}\right)+\epsilon>f\left(X^{*}\right)
\end{aligned}
$$

which contradicts the fact that $X^{*}$ is an optimal solution for the penalized formulation. Therefore, $x^{*}$ is a feasible solution for the SCP formulation. In a second step, we prove that this truncated solution $x^{*}$ is optimal for the SCP formulation. We show this again by contradiction: suppose that $x^{*}$ were not an optimal solution for the SCP formulation; then, there exists $\hat{x}$ feasible for SCP that is better than $x^{*}$ for $\mathrm{SCP}$, i.e. such that $\sum_{j=1}^{m} \hat{x}_{j}<\sum_{j=1}^{m} x_{i}^{*}$. Consider now the following solution $\hat{X}$ for the penalized formulation: $\hat{X}:=(\hat{x}, \hat{y})$, where $\hat{y}_{i}:=\min \left(\sum_{j \in S_{i}} \hat{x}_{j}, K\right)$. We have:

$$
\begin{aligned}
f(\hat{X})-f\left(X^{*}\right)= & \left(\sum_{i=1}^{n}(1+\epsilon) \hat{y}_{i}-\sum_{j=1}^{m} \hat{x}_{j}\right)-\left(\sum_{i=1}^{n}(1+\epsilon) y_{i}^{*}-\sum_{j=1}^{m} x_{j}^{*}\right) \\
= & \left(\sum_{i=1}^{n}(1+\epsilon) K-\sum_{j=1}^{m} \hat{x}_{j}\right)-\left(\sum_{i=1}^{n}(1+\epsilon) K-\sum_{j=1}^{m} x_{j}^{*}\right) \\
& \quad \text { since both } \hat{x} \text { and } x^{*} \text { satisfy }(2) \text { and therefore } \hat{y}_{i}=y_{i}^{*}=K \\
= & \sum_{j=1}^{m} x_{j}^{*}-\sum_{j=1}^{m} \hat{x}_{j} \\
> & \text { since } \hat{x} \text { is better than } x^{*} \text { for the SCP formulation. }
\end{aligned}
$$

This means that $\hat{X}$ is strictly better than $X^{*}$ in terms of the penalized objective function, and since moreover $\hat{X}$ is feasible for the penalized formulation, we obtain a contradiction with the fact that $X^{*}$ is an optimal solution for the penalized formulation. Therefore, $x^{*}$ is an optimal solution for the SCP formulation.

To show the converse, let us consider a solution $x^{*}$ that is optimal for the SCP formulation. We shall show that the extended solution: $X^{*}:=\left(x^{*}, y^{*}\right)$, where $y_{i}^{*}:=\min \left(\sum_{j \in S_{i}} x_{j}^{*}, K\right)=K$ is optimal for the penalized formulation. This solution $x^{*}$ is clearly feasible for the penalized formulation. 
Consider now any solution $X=(x, y)$ satisfying (5) and (6). We have:

$$
\begin{aligned}
f\left(X^{*}\right)-f(X)= & \left(\sum_{i=1}^{n}(1+\epsilon) y_{i}^{*}-\sum_{j=1}^{m} x_{j}^{*}\right)-\left(\sum_{i=1}^{n}(1+\epsilon) y_{i}-\sum_{j=1}^{m} x_{j}\right) \\
\geq & \left.\sum_{i=1}^{n}(1+\epsilon) K-\sum_{j=1}^{m} x_{j}^{*}-\sum_{i=1}^{n}(1+\epsilon) K+\sum_{j=1}^{m} x_{j}\right) \\
& \quad \text { since for all } i: y_{i}^{*}=K \text { while } y_{i} \leq K \\
= & \sum_{j=1}^{m} x_{j}-\sum_{j=1}^{m} x_{j}^{*} \\
\geq & 0 \quad \text { since } x^{*} \text { is optimal for the SCP formulation }
\end{aligned}
$$

Therefore, $X^{*}$ is optimal for the penalized formulation.

\section{References}

[1] Gurobi Optimization I, Gurobi optimizer reference manual, 2017.

[2] N. Bilal, P. Galinier, and F. Guibault. A new formulation of the set covering problem for metaheuristic approaches. ISRN Operations Research, 2013.

[3] E. S. Boci. RF coverage analysis methodology as applied to ADS-B design. In 2009 IEEE Aerospace Conference, Big Sky, MT, United States, Mar 2009.

[4] E. S. Boci. A systems approach for designing a radio station layout for the U.S. national airspace. $\mathrm{PhD}$ thesis, The George Washington University, 2010.

[5] E. S. Boci, S. Sarkani, and T. Mazzuchi. ADS-B service volume engineering: Designing an efficient radio station layout while meeting stringent radar interference levels. International Journal of Mobile Network Design and Innovation, 3(3), 2010.

[6] M. Daskin. Introduction to Location Theory and Models, chapter 1, pages 1-19. Wiley-Blackwell, 2011.

[7] J. Guan, Z. G. Su, and H. Y. Wu. SSR surveillance network covering problem with obstacle effect consideration. Advanced Materials Research, 926-930:2097-2101, 2014.

[8] W. Langhans, C. Scheiflinger, W. Weidner, J. Auer, P. Fitzgerald, and M. Anzalone. Implementation of a nationwide wide-area multilateration system for Austrian airspace. In Integrated Communications, Navigation and Surveillance Conference (ICNS), Virginia, United States, Apr 2013.

[9] R. Minciardi, R. Sacile, and F. Siccardi. Optimal planning of a weather radar network. Journal of Atmospheric and Oceanic Technology, 20(9):1251-1263, 2003. 
[10] W. Zhang. The research of ADS-B ground stations arrangement based on GIS. Master's thesis, Civil Aviation Flight University of China, Guanghan, 2014. 